\title{
Optimization of Well Placement and Production for Large-scale Mature Oil Fields
}

\author{
Xiang Wang ${ }^{1}$, Qihong Feng ${ }^{1 *}$ and Ronald D. Haynes ${ }^{2}$ \\ ${ }^{1}$ School of Petroleum Engineering, China University of Petroleum (Huadong), Qingdao, Shandong, 266580, China \\ ${ }^{2}$ Department of Mathematics \& Statistics, Memorial University of Newfoundland, St. John's, NL, A1C 5S7, Canada
}

Received 18 June 2015; Accepted 11 December 2015

\begin{abstract}
Optimal oil field development strategies, especially well locations and production strategies for mature oil fields, should be determined to sustain yields. For a large-scale oil field, these problems are nonlinear, nonconvex, and computationally expensive. In this study, an efficient and robust derivative-free computational framework was developed to determine the optimal number, locations, and injection/production rates of infill wells for mature oil fields. The characteristics of mature fields were briefly described; optimization formulation and computational framework were presented. For this problem, the robust and parallelizable PSwarm, a hybrid of a pattern search algorithm and a particle swarm optimization, was investigated. The approach was applied to a large-scale real oil field that currently includes approximately 200 wells. Our optimized results were compared with those of the current plan provided by the oil industry. In particular, a higher oil production with the same amount of water injection and a higher net present value were obtained by our optimized approach than by the current plan. Therefore, the new derivative-free computational framework can efficiently solve well placement and production optimization problems for large-scale mature oil fields.
\end{abstract}

Keywords: Well placement, Well production, Mature oil field, Derivative-free optimization, Reservoir simulation-based optimization

\section{Introduction}

Well placement and production optimization have been extensively investigated. However, few research efforts have focused on mature oil fields. A mature field is a field that has been developed for many years ( $>10$ years); this field is also characterized by a high water cut $(>75 \%)$ [1]. These fields have considerable potential because they have generally been developed for many years under suboptimal conditions. Development adjustments, such as drilling new infill wells and changing the injection/production rates of wells, can improve the performance of mature fields and significantly increase oil recovery [2]. Infill wells are new wells added to an existing field within original well patterns. Infill drilling can accelerate oil recovery in heterogeneous reservoirs. Shifting well patterns alter fluid flow paths and increase sweep to areas with high oil saturations because well spacing is decreased. Nevertheless, this adjustment plan is difficult to distinguish because reservoir performance is affected by geological, engineering, economical, and other parameters. These problems can be formulated as several distinct optimization problems or as a joint optimization problem.

Previous studies addressed the separate optimization of well locations [3, 4], well controls [5-7], and joint optimization of well location and controls [8]. Few studies have focused on the optimization of the number of new wells combined with well location and control optimization [9]. In this work, the optimization problems related to

* E-mail address: fengqihong@126.com ISSN: 1791-2377 @ 2015 Kavala Institute of Technology. All rights reserved. mature fields were considered. These problems include the optimization of the number of infill wells, the locations of infill wells, and the injection/production rate of all wells. These optimization problems can be solved either simultaneously or sequentially $[8,10]$. Although simultaneous problems include global optima, a high computational budget is required and algorithms pose an increased risk of falling into local optima because of the large number of optimization variables. The sequential approach decouples the joint problem into several subproblems; as a result, problems can be solved more easily with optimized strategies than with existing approaches.

Gradient-based algorithms and derivative-free algorithms have been investigated to solve well placement and production optimization problems. The gradient information of the objective function is employed by gradient-based algorithms [11, 12] in searching for optimal solutions, such as the steepest ascent method and the simultaneous perturbation stochastic approximation algorithm. For well placement and control optimization, a solution surface is generally nonconvex and has discontinuities. Thus, gradients are not easily calculated. Derivative-free algorithms [9, 13, 14], such as generalized pattern search and particle swarm optimization, are generally robust and naturally parallel; these algorithms have been commonly applied to solve well placement and control optimization problems.

Considering the characteristics and the need for mature fields, we develop a framework to determine the optimal number of infill wells, their locations, and the optimal injection/production rates of all wells. As a global derivative-free optimization algorithm combining pattern search and particle swarm, PSwarm $[15,16]$ is applied as a 
solver in the framework. This algorithm is robust and efficient for smooth and non-smooth problems in serial way and in parallel way $[16,17]$. Our framework is applied to a large-scale real field that includes approximately 200 wells. The results are compared with those of a current plan provided by the oil industry.

This paper is organized as follows. Section 2 describes the formulation of well placement and production optimization problems. Section 3 discusses the framework used to solve these problems. Section 4 provides an overview of PSwarm. Section 5 introduces our test case. Section 6 presents our conclusions.

\section{Problem formulation}

The generalized well placement and production optimization problem for mature fields can be stated as follows:

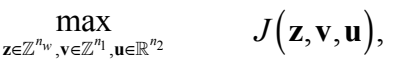

$$
\begin{aligned}
& \text { subject to } \mathbf{g}(\mathbf{z}, \mathbf{v}, \mathbf{u})=\mathbf{0} \text {, } \\
& \mathbf{c}(\mathbf{z}, \mathbf{v}, \mathbf{u}) \leq \mathbf{0} \text {, }
\end{aligned}
$$

where $J$ is the objective function, either the net present value (NPV) or the cumulative oil produced; $\mathbf{g}$ and $\mathbf{c}$ are equality and inequality constraints, respectively. $\mathbf{z}$ denotes the number of the infill wells; $\mathbf{v}$ denotes the location variables of the infill wells; and $\mathbf{u}$ denotes the well production variables, which are either bottom hole pressures or injection/production rates.

The total number of variables depends on the number of infill wells. If the number of infill wells is $N_{\text {infill }}$, and all infill wells are vertical, the locations of each well are provided by plane coordinates $(x, y)$. Hence, the total number of variables for the generalized problem is $2 N_{\text {infill }}+\left(N_{\text {exist }}+N_{\text {infill }}\right)$, where $N_{\text {exist }}$ is the number of existing wells. $n_{1}=2 N_{\text {infill }}$ variables are associated with well placements and $n_{2}=N_{\text {exist }}+N_{\text {infill }}$ variables are associated with well production.

In this work, NPV is considered as the objective function. The optimization problem can be represented as follows:

$$
\begin{gathered}
\max _{\mathbf{z} \in \mathfrak{d}, \mathbf{v} \in \mathbb{t}, \mathbf{u} \in^{\circ}}-\sum_{i=1}^{N_{\text {iniux }}^{\max }} z_{i} C_{i}+\sum_{k=1}^{N_{t}} \frac{\Delta t_{k}}{(1+b)^{t_{k} / \tau}} \\
{\left[\sum_{i=1}^{N_{\text {infiul }}^{\max }}\left(r^{p o} q_{i, k}^{p o}(\mathbf{u}, \mathbf{v})-c^{p w} q_{i, k}^{p w}(\mathbf{u}, \mathbf{v})-c^{i w} q_{i, k}^{i w}(\mathbf{u}, \mathbf{v})\right)\right.} \\
\left.+\sum_{i=1}^{N_{\text {exist }}}\left(r^{p o} q_{i, k}^{p o}(\mathbf{u})-c^{p w} q_{i, k}^{p w}(\mathbf{u})-c^{i w} q_{i, k}^{i w}(\mathbf{u})\right)\right] \\
\text { subject to } \quad \mathbf{u}_{\mathrm{lb}} \leq \mathbf{u} \leq \mathbf{u}_{\mathrm{ub}}, \\
\mathbf{v}_{\mathrm{lb}} \leq \mathbf{v} \leq \mathbf{v}_{\mathrm{ub}}, \\
\sum_{i=1}^{N_{\text {infiul }}^{\max }} z_{i} \leq K_{\max }, \\
z_{i} \in\{0,1\} \quad i=1, \mathrm{~L}, K_{\max }
\end{gathered}
$$

where $N_{\text {infill }}^{\max }$ is the maximum number of infill wells to be drilled; $z_{i}$ is a binary variable that prescribes whether or not a well is drilled; $C_{j}$ is the cost of drilling the infill well $i$; $N_{t}$ and $\Delta t_{k}$ denote the number of time steps and step size at step $k$, respectively; $r^{p o}$ is the produced oil revenue; and $c^{p w}$ and $c^{i w}$ are the cost of dealing with water produced and the cost of injected water. $q_{i, k}^{p o}, q_{i, k}^{p w}$, and $q_{i, k}^{i w}$ are the flow rates of oil, water produced, and water injected into well $i$ at time step $k$, respectively. The time at the end of $k$ th time step is $t_{k} ; \tau=365$ days provides the appropriate normalization for time $t_{k}$; and $b$ is the fractional discount rate.

$q_{i, k}^{p o}, q_{i, k}^{p w}$, and $q_{i, k}^{i w}$ are functions of the well locations and rates; these variables can be obtained from a reservoir simulator. The constraints considered in this problem include bound constraints for well locations, bound constraints for production rates, and a maximum number of infill wells.

\section{Optimization framework}

The generalized optimization problem for mature fields includes integer variables (the number of infill wells and the infill well locations) and continuous variables (well rates). The total number of variables can be extremely large and thus can cause a large, high-dimensional search space. Moreover, the search space becomes discontinuous and rough because of the integer variables. However, the optimization of these variables requires a high computational budget; as such, this process is impractical for many applications.

On the basis of this situation, we propose a computational framework that decouples the generalized optimization problem into three subproblems. We then optimize these three subproblems sequentially. The subproblems include the optimization of the number of infill wells, the optimization of the locations of infill wells, and the optimization of well production. The search space of each subproblem is significantly smaller than that of the joint problem. Although the global optima cannot be theoretically obtained, the sequential procedures reduce computational load. Thus, the optimal number, locations, and rates of wells for large-scale mature fields can be determined.

\subsection{Optimizing the number of infill wells}

The optimization of the number of infill wells is challenging because the optimal number of infill wells depends on the locations of the infill wells and how the well is operated. Instead of a numerical optimization method, a reservoir engineering theory is applied to determine the reasonable well density and to calculate the number of infill wells needed for the reservoir.

According to reservoir engineering theory $[18,19]$, the relationship between the ultimate oil recovery and the well density can be stated as follows:

$\eta=\eta_{o} e^{-a S_{a}^{-1}}$

where $S_{a}$ is the well density, $\eta$ is the ultimate recovery, $\eta_{o}$ is the displacement efficiency, and $a$ is thewell-pattern coefficient. 
From economic considerations [18], the reasonable well density $S_{r}$ is given as follows:

$a S_{r}^{-1}=\ln \frac{N k \eta_{o} a}{F C}+2 \ln S_{r}^{-1}$

where $N$ is the geological reserves of the reservoir, $k$ is the sale price of oil, $F$ is the reservoir area, and $C$ is the cost of drilling each well.

The optimal number of infill wells $N_{\text {infill }}^{\text {opt }}$ can be obtained by comparing the reasonable well density $S_{r}$ and the actual well density $S_{a}$ :

$N_{\text {infill }}^{\mathrm{opt}}=F\left(S_{r}-S_{a}\right)$.

For a mature reservoir, $S_{a}, \eta, \eta_{o}, N, k, F$, and $C$ are known. We initially determine the value of constant $a$ by using Equation (3). Then, we calculate the reasonable well density $S_{r}$ by solving Equation (4). The number of infill wells can be obtained through Equation (5).

Infill wells include production wells and injection wells. The number of infill production wells and the number of infill injection wells are related by the following equation:

$R=\frac{N_{\text {exist }, \mathrm{o}}+N_{\text {infill,o }}}{N_{\text {exist, } \mathrm{w}}+N_{\text {infill, } \mathrm{w}}}$,

where $N_{\text {exist,o }}, N_{\text {exist,w }}, N_{\text {infill,o }}$, and $N_{\text {infill,w }}$ are the number of existing production wells, the number of existing injection wells, the number of infill production wells, and the number of infill injection wells, respectively. $R$ is the reasonable production-injection well ratio, usually provided by the oil industry.

\subsection{Optimizing the locations of infill wells}

In the well placement optimization problem, we seek to determine the optimal locations for a specified number of infill wells. The optimization problem is defined as follows:

$$
\begin{aligned}
& \max _{\mathbf{v} \in \mathbb{\mathfrak { t }}} \sum_{k=1}^{N_{t}} \frac{\Delta t_{k}}{(1+b)^{t_{k} / \tau}} \\
& {\left[\sum_{i=1}^{N_{\text {inftul }}^{\text {opt }}}\left(r^{p o} q_{i, k}^{p o}(\mathbf{v})-c^{p w} q_{i, k}^{p w}(\mathbf{v})-c^{i w} q_{i, k}^{i w}(\mathbf{v})\right)\right.} \\
& \left.+\sum_{i=1}^{N_{\text {exist }}}\left(r^{p o} q_{i, k}^{p o}-c^{p w} q_{i, k}^{p w}-c^{i w} q_{i, k}^{i w}\right)\right] \\
& \text { subject to } \quad \mathbf{v}_{\mathrm{lb}} \leq \mathbf{v} \leq \mathbf{v}_{\mathrm{ub}} .
\end{aligned}
$$

During the well placement optimization procedure, all wells are produced with specified reasonable production rates.

\subsection{Optimizing the well production}

The well production optimization problem aims to determine the optimal rates for each of the production and injection wells. The optimization problem can be stated as follows:

$$
\begin{aligned}
& \max _{\mathbf{u} \in{ }^{\circ}} \sum_{k=1}^{N_{t}} \frac{\Delta t_{k}}{(1+b)^{t_{k} / \tau}} \\
& {\left[\sum_{i=1}^{N_{\text {infilu }}^{\text {opt }}}\left(r^{p o} q_{i, k}^{p o}(\mathbf{u})-c^{p w} q_{i, k}^{p w}(\mathbf{u})-c^{i w} q_{i, k}^{i w}(\mathbf{u})\right)\right.} \\
& \left.+\sum_{i=1}^{N_{\text {cxist }}}\left(r^{p o} q_{i, k}^{p o}(\mathbf{u})-c^{p w} q_{i, k}^{p w}(\mathbf{u})-c^{i w} q_{i, k}^{i w}(\mathbf{u})\right)\right], \\
& \text { subject to } \quad \mathbf{u}_{\mathrm{lb}} \leq \mathbf{u} \leq \mathbf{u}_{\mathrm{ub}} .
\end{aligned}
$$

\section{Optimization algorithm PSwarm}

In this section, we briefly describe the optimization algorithm considered in this study. PSwarm [15] is a hybrid solver for global derivative-free optimization. By combining a pattern search method and a particle swarm search algorithm, PSwarm performs a heuristic search (particle swarm) for the global optimization with a rigorous method (pattern search) for the local optimization. Hence, PSwarm exhibits global convergence properties and can guarantee convergence to stationary point [16]. Moreover, PSwarm is a derivative-free algorithm especially suitable for practical instances where derivatives are unknown, or where derivatives are noisy and meaningless.

PSwarm first performs particle swarm optimization. Whenever the particle swarm optimization fails to find a better solution, a local search is performed. During the local search phase, the poll step is applied to the best particle. The detailed description for PSwarm is provided in Algorithm 1.

Algorithm 1. PSwarm algorithm [15].

a. Set the stopping tolerances $\alpha_{\text {tol }}>0, v_{\text {tol }}>0$.

b. Set the initial population size $s$, let $\mathrm{I}=\{1, \mathrm{~L}, s\}$.

c. Calculate the initial feasible swarm positions $x_{0}^{1}, \mathrm{~L}, x_{0}^{s}$.

d. Calculate the initial swarm velocities $v_{0}^{1}, \mathrm{~L}, v_{0}^{s}$.

e. Set $y_{0}^{i}=x_{0}^{i}, i=1, \mathrm{~L}, s, \hat{y}_{0} \in \arg \min _{z \in\left\{y_{0}^{1} \mathrm{~L}, y_{0}^{s}\right\}} f(z)$.

f. Choose $\alpha_{0}>0$, let $t=0$.

\section{g. [Search step]}

Set $\hat{y}_{t+1}=\hat{y}_{t}$.

for all $i \in \mathrm{I}$, do:

$$
\begin{aligned}
& \text { if } f\left(x_{t}^{i}\right)<f\left(y_{t}^{i}\right) \text {, then } \\
& \text { Set } y_{t+1}^{i}=x_{t}^{i} . \\
& \text { if } y_{t+1}^{i}<\hat{y}_{t+1} \text {, then } \\
& \text { Set } \hat{y}_{t+1}=y_{t+1}^{i} . \\
& \text { Set } \alpha_{t+1}=\phi_{t} \alpha_{t} . \\
& \text { end if }
\end{aligned}
$$

else

$$
\text { Set } y_{t+1}^{i}=y_{t}^{i} \text {. }
$$

end if

\section{end for}

h. [Poll step]

if the search step was successful, then

Skip the poll step. else

if there exists $d(t) \in D$ s.t. $\hat{f}\left(\hat{y}_{t}+\alpha_{t} d_{t}\right)<\hat{f}\left(\hat{y}_{t}\right)$

Set $\hat{y}_{t+1}=\hat{y}_{t}+\alpha_{t} d_{t}$

Set $\alpha_{t+1}=\phi_{t} \alpha_{t}$. 
else if $\hat{f}\left(\hat{y}_{t}+\alpha_{t} d_{t}\right) \geq \hat{f}\left(\hat{y}_{t}\right)$, then

Set $\hat{y}_{t+1}=\hat{y}_{t}$. end if

Set $\alpha_{t+1}=\theta_{t} \alpha_{t}$.

end if

i. Compute $v_{t+1}^{i}=l_{t} v_{t}^{i}+\mu \omega_{1, t}\left(y_{t}^{i}-x_{t}^{i}\right)+v \omega_{2, t}\left(\hat{y}_{t}-x_{t}^{i}\right), i \in \mathrm{I}$.

j. Compute $x_{t+1}^{i}=x_{t}^{i}+\alpha_{\max ^{i}} v_{t+1}^{i}, i \in \mathrm{I}$.

k. if $\alpha_{t+1}^{i}<\alpha_{\text {tol }}$ and $\left\|v_{t+1}^{i}\right\|<v_{\text {tol }}, i=1, \mathrm{~L}, s$,

Stop.

else

Let $t=t+1$.

Drop particles in the search step if too close to each other, update I .

Go to Step g.

end if

Our implementation of PSwarm uses the parameters given in the work of $\mathrm{Vaz}$ et al. [15]. We choose $\phi_{t}=1$, $\theta_{t}=0.5$. After two consecutive poll successes along the same direction, we choose $\phi_{t}=2$. The other parameters are chosen as $v=\mu=0.5 ; s=20 ; l_{t}=0.9-\left(0.5 / t_{\max }\right) t$, where $t_{\max }$ is the maximum number of iterations.

\section{Example case}

\subsection{Reservoir description}

A full-scale mature oil field is considered in this work. The geological and flow properties of the reservoir are provided in Table 1. The relative permeability curves are shown in Figure 1. The reservoir has been developed under water flooding for 50 years. This reservoir is in an extra high water cut stage with a water cut of $95.6 \%$. The number of active production wells is 82 and the number of active injection wells is 64 . Figure 2 shows the permeability, porosity, and present oil saturation, together with the locations of all wells in the reservoir.

Table. 1. Geological and flow properties of the reservoir.

\begin{tabular}{lcc}
\hline Property & Values & Units \\
\hline Oil bearing area & 19.1 & $\mathrm{~km}^{2}$ \\
Average porosity & 0.3 & - \\
Permeability & $100-2800$ & $10^{3} \mathrm{\mu m}^{2}$ \\
Geological reserves & 3740 & $10^{4} \mathrm{t}$ \\
Oil viscosity in place & $10-26$ & $\mathrm{mPa} \cdot \mathrm{s}$ \\
Oil density in place & $0.84-0.88$ & $\mathrm{~g} / \mathrm{cm}^{3}$ \\
Initial pressure & 11.9 & $\mathrm{MPa}$ \\
Initial temperature & 80 & ${ }^{\circ} \mathrm{C}$ \\
Reservoir buried depth & $1890-2120$ & $\mathrm{M}$ \\
\hline
\end{tabular}

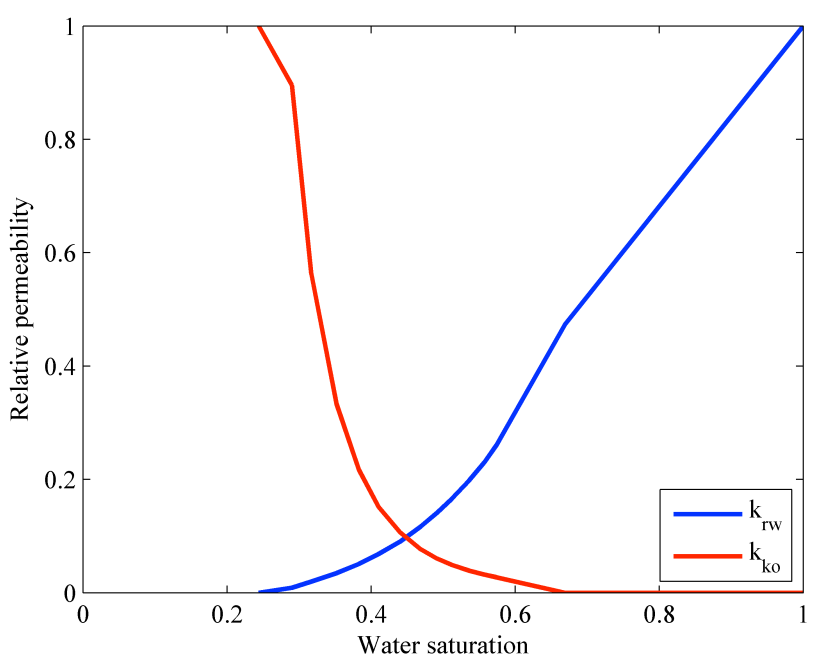

Fig. 1. Relative permeability curves of the reservoir.

The simulation model of the reservoir contains a twophase oil-water system with $238 \times 161 \times 13$ grid blocks; of these blocks, 127,665 are active. We use a production period of 15 years for the optimization. The economic parameters and additional optimization settings are listed in Table 2.

Table 2. Economic parameters and additional optimization settings for the reservoir.

\begin{tabular}{lcc}
\hline Property & Values & $\mathrm{Units}$ \\
\hline Oil revenue & 500 & $\mathrm{USD} / \mathrm{m}^{3}$ \\
Water production cost & 10 & $\mathrm{USD} / \mathrm{m}^{3}$ \\
Water injection cost & 10 & $\mathrm{USD} / \mathrm{m}^{3}$ \\
Well drilling cost & 40000 & $\mathrm{USD} / \mathrm{well}$ \\
Maximum production rate & 120 & $\mathrm{~m} / \mathrm{d}$ \\
Maximum injection rate & 200 & $\mathrm{~m}^{3} / \mathrm{d}$ \\
\hline
\end{tabular}

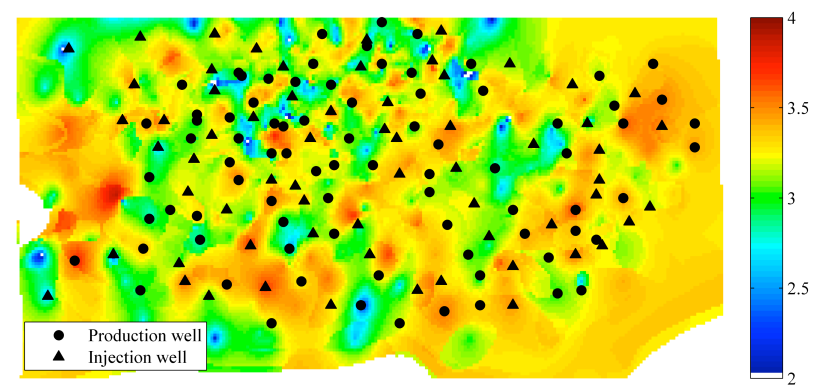

(a) $\log$ (Permeability), $10^{3} \mu \mathrm{m}^{2}$

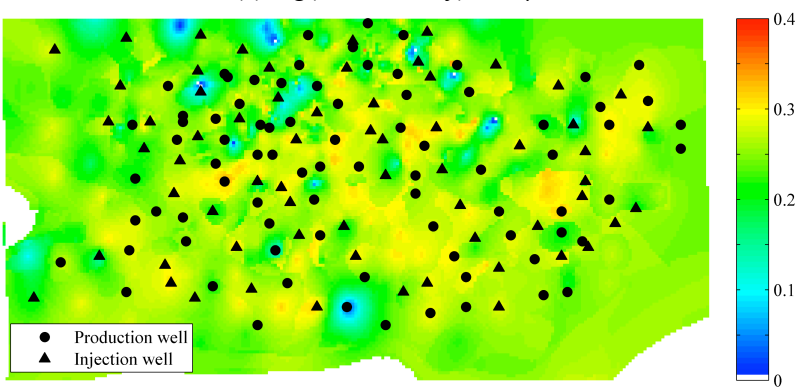

(b) Porosity 


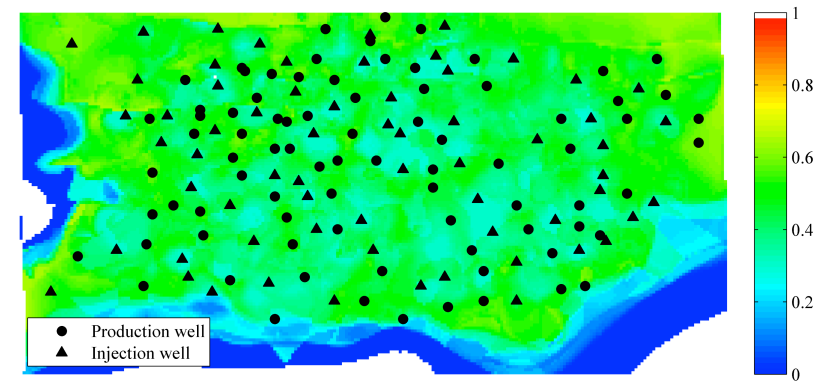

(c) Present oil saturation

Fig. 2. Properties and wells of the mature reservoir.

\subsection{Results and Discussion}

The optimal number of infill wells, together with their locations, and the optimal injection/production rates of all wells are calculated by using the proposed framework.

For the reservoir, the number of existing wells $N_{\text {exist }}$ is 146. The current well density $S_{a}$ is 7.64 well $/ \mathrm{km}^{2}$. The ultimate recovery $\eta$ is 0.4971 and the displacement efficiency $\eta_{o}$ is 0.4982 . According to Equation (3),

$$
0.4971=0.4982 e^{-a \times 7.64^{-1}},
$$

and thus $a=0.0065$.

Substituting all the known parameters into Equation (4), we derive the following:

$$
\begin{gathered}
0.0065 S_{r}^{-1}= \\
\ln \frac{3740 \times 10^{4} \times 500 \times 0.4982 \times 0.0065}{19.1 \times 4 \times 10^{4}}+2 \ln S_{r}^{-1} .
\end{gathered}
$$

This equation provides the reasonable well density $S_{r}$ of $8.90 \mathrm{well} / \mathrm{km}^{2}$.

The number of infill wells can then be calculated according to Equation (5) as follows:

$$
N_{\text {infill }}^{\text {opt }}=19.1 \times(8.90-7.64)=24 \text {. }
$$

The reasonable production-injection well ratio is given $R=1.21$, as provided by the oil industry. We also find that the number of existing production wells $N_{\text {exist, }}=82$ and the number of existing injection wells $N_{\text {exist,w }}=64$. Substituting these parameters into Equation (6), we obtain the following relation:

$$
1.21=\frac{82+N_{\text {infill,o }}}{64+N_{\text {infill,w }}} .
$$

Considering that $N_{\text {infill, }}+N_{\text {infill, }}=N_{\text {infill }}^{\text {opt }}=24$, we can calculate the number of infill production wells, $N_{\text {infill,o }}=11$ and the number of infill injection wells, $N_{\text {infill,w }}=13$.

The optimized locations of infill wells were obtained by the well placement optimization phase of the framework as described in Section 3, and are provided in Figure 3, in which, black circles and triangles denote the existing production and injection wells, respectively. Red circles and triangles denote the infill production wells and infill injection wells, respectively. The base map is current oil saturation. The detailed location information of infill wells is shown in Table 3. The average distance between the artificially designed locations of infill wells and the optimized locations of infill wells is $84 \mathrm{~m}$.

The well rates were optimized by the well production optimization phase of the framework by solving the problem prescribed in Section 3. A visual comparison between the initial well rates and the optimized well rates is shown in Figure 4, where magenta and red bars denote the initial and optimized production rates of all the production wells, respectively. Cyan and blue bars denote the initial and optimized injection rates of all injection wells, respectively. The base map is current oil saturation.

Figure 5 shows the performance of PSwarm algorithm for the well placement optimization for the placement of the infill wells and the well production optimization of all wells, respectively. PSwarm algorithm can solve this kind of largescale constrained optimization problem.

The initial guesses for these two optimization problems are obtained from the adjustment plan provided by the oil industry. Figure 5(a) reveals that the traditional adjustment plan design given by petroleum engineers for this reservoir cannot ensure a good development output because of the extreme high water cut. For this mature field, the NPV is less than 0 when the unoptimized adjustment plan is used. The NPV significantly increases after well placement

\begin{tabular}{|c|c|c|c|c|c|}
\hline Infill well index & I, original & $\mathbf{J}$, original & I, optimized & J, optimized & Distance, $\mathbf{m}$ \\
\hline 1 & 217 & 67 & 216 & 66 & 42.43 \\
\hline 2 & 218 & 78 & 217 & 75 & 94.87 \\
\hline 3 & 206 & 64 & 205 & 63 & 42.43 \\
\hline 4 & 207 & 72 & 209 & 70 & 84.85 \\
\hline 5 & 175 & 43 & 178 & 42 & 94.87 \\
\hline 6 & 186 & 76 & 183 & 73 & 127.28 \\
\hline 7 & 166 & 54 & 168 & 52 & 84.85 \\
\hline 8 & 174 & 75 & 173 & 72 & 94.87 \\
\hline 9 & 159 & 62 & 158 & 60 & 67.08 \\
\hline 10 & 147 & 49 & 144 & 49 & 90.00 \\
\hline 11 & 138 & 100 & 138 & 98 & 60.00 \\
\hline 12 & 134 & 88 & 131 & 85 & 127.28 \\
\hline 13 & 126 & 96 & 126 & 93 & 90.00 \\
\hline 14 & 125 & 83 & 124 & 86 & 94.87 \\
\hline 15 & 116 & 81 & 114 & 82 & 67.08 \\
\hline 16 & 103 & 105 & 105 & 104 & 67.08 \\
\hline
\end{tabular}
optimization and well production optimization are completed. The plot of cumulative oil production versus time is illustrated in Figure 6.

Table 3. Artificially designed infill well locations and optimized locations. 


\begin{tabular}{cccccc}
17 & 109 & 31 & 112 & 29 & 108.17 \\
18 & 52 & 93 & 50 & 91 & 27 \\
19 & 58 & 27 & 56 & 32 & 60.00 \\
20 & 186 & 34 & 188 & 32 & 84.85 \\
21 & 173 & 30 & 176 & 61 & 108.17 \\
22 & 23 & 58 & 23 & 64 & 90.00 \\
23 & 78 & 66 & 80 & 44 & 84.85 \\
24 & 26 & 43 & 24 & & 67.08 \\
\hline
\end{tabular}

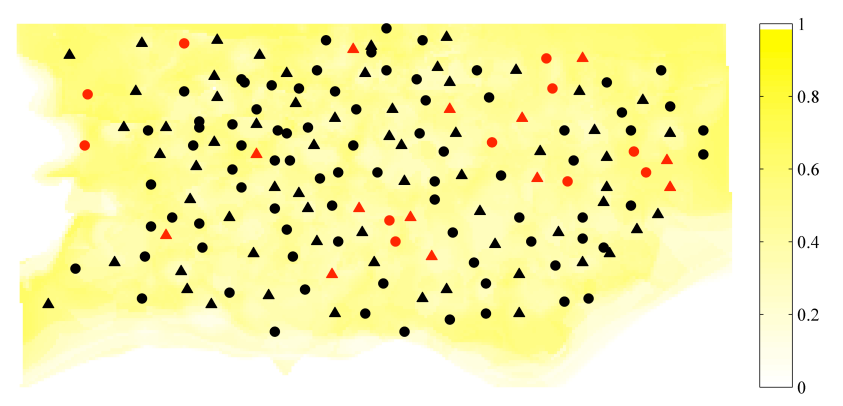

Fig. 3. Optimized locations of infill wells for the reservoir.

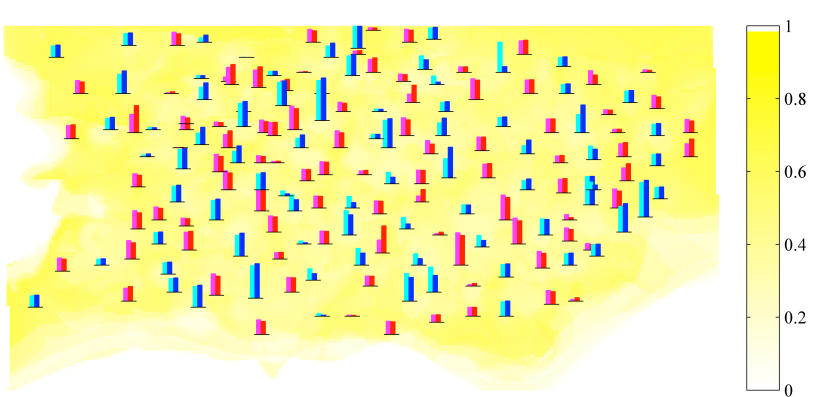

Fig. 4. Initial rates and optimized rates of all wells in the reservoir.

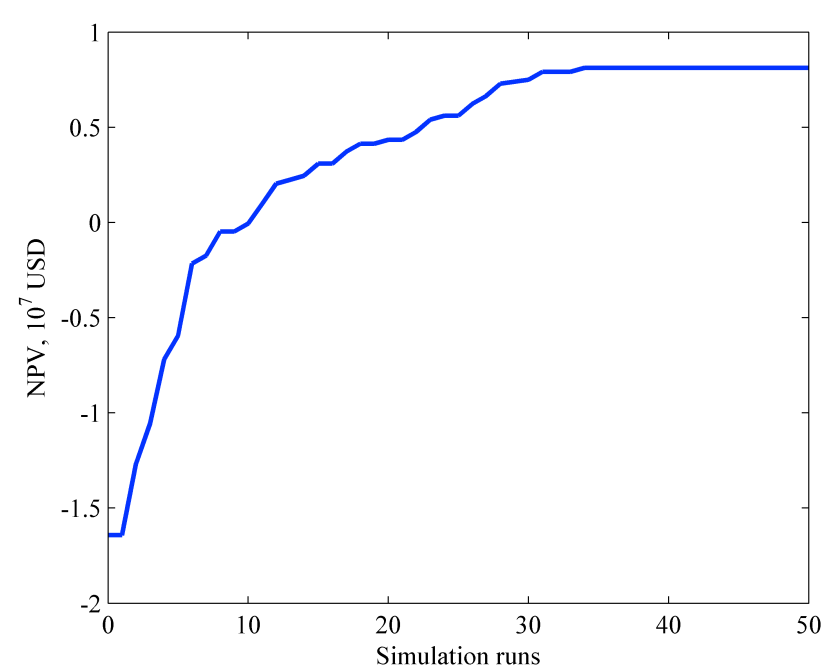

(a) well placement optimization

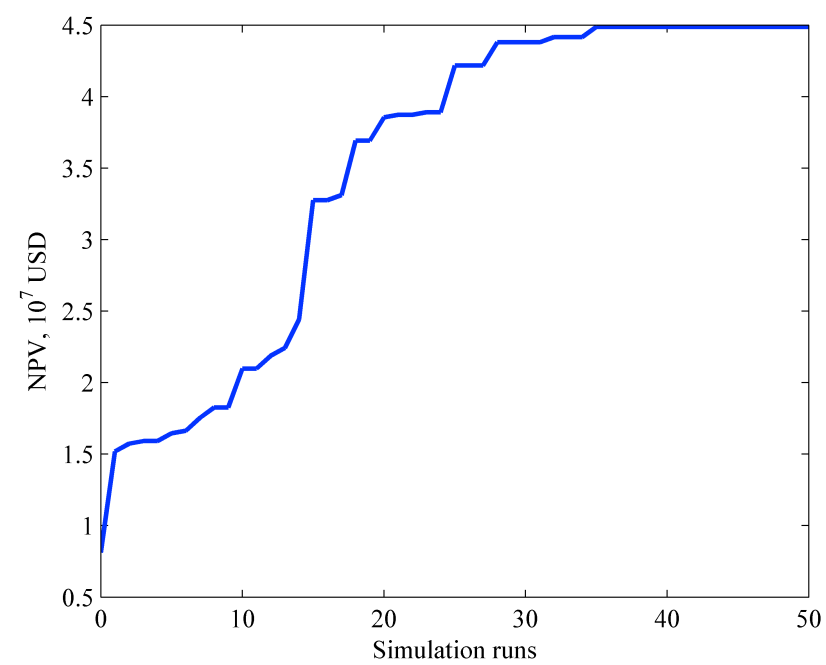

(b) well production optimization

Fig. 5. Performance of PSwarm algorithm for the well placement optimization of the infill wells and the well production optimization of all wells.

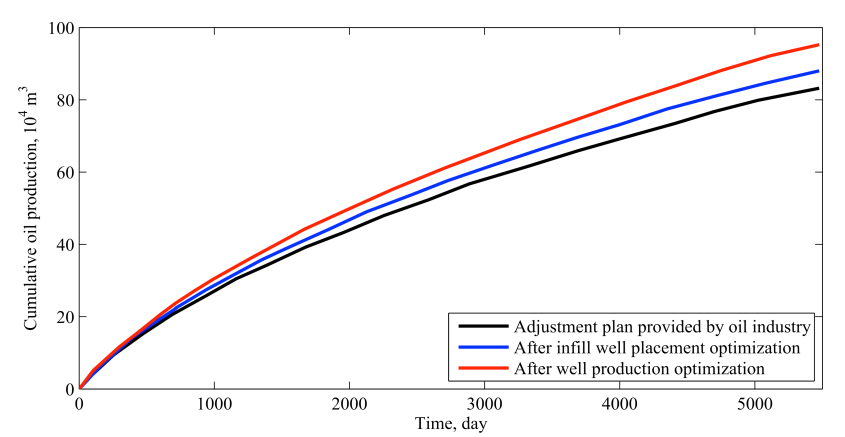

Fig. 6. Plot of cumulative oil production versus time.

\section{Conclusions}

In this study, a computational framework was introduced to optimize the number of infill wells, the location of infill wells, and the production of all wells for a large-scale mature field. The framework decouples the joint complex optimization problem into three individual subproblems and solves these subproblems sequentially. This decoupling decreases the problem difficulty; as a result, optimization can be achieved for a large-scale field containing hundreds of wells. We also proposed the PSwarm algorithm, an efficient and robust derivative-free method that combines a pattern search and particle swarm optimization as the optimizer within the computational framework. The framework is applied to a full-scale mature oil field that includes nearly 200 wells. The results are compared with a current plan provided by the oil industry. The plan found by the optimization approach can obtain higher NPV and oil production than the current plan provided by the oil industry. 
The computational framework has shown its potential in our work, but further research should be conducted. Future work should focus on exploring the performance of computational frameworks while iterating well placement optimization procedure and well control optimization procedure. Further studies should also simultaneously optimize the number of infill wells, the location of infill wells, and the control of all wells.

\section{Acknowledgements}

The study was supported by the Natural Sciences and Engineering Research Council of Canada (NSERC) Discovery Grant Program, the National Science and Technology Major Project of the Ministry of Science and Technology of China (2011ZX05011-002), and the program of China Scholarships Council (No. 201406450017).

\section{References}

1. Wen, T., Thiele, M. R., Ciaurri, D. E., Aziz, K., Ye, Y., "Waterflood management using two-stage optimization with streamline simulation". Computational Geosciences, 18 (3-4), 2014, pp.483-504.

2. Taware, S. V., Park, H.-y., Datta-Gupta, A., Bhattacharya, S., Tomar, A. K., Kumar, M., Rao, H. S., "Well placement optimization in a mature carbonate waterflood using streamlinebased quality maps". in: SPE Oil and Gas India Conference and Exhibition, Society of Petroleum Engineers, 2012.

3. Bouzarkouna, Z., Ding, D. Y., Auger, A., "Well placement optimization with the covariance matrix adaptation evolution strategy and meta-models". Computational Geosciences, 16 (1), 2012, pp.75-92.

4. AlQahtani, G. D., Alzahabi, A., Spinner, T., Soliman, M. Y., "A Computational Comparison between Optimization Techniques for Wells Placement Problem: Mathematical Formulations, Genetic Algorithms and Very Fast Simulated Annealing". Journal of Materials Science and Chemical Engineering, 2 (10), 2014, pp.5973.

5. Asadollahi, M., Nævdal, G., Dadashpour, M., Kleppe, J., "Production optimization using derivative free methods applied to Brugge field case". Journal of Petroleum Science and Engineering, 114, 2014, pp.22-37.

6. Oliveira, D. F., Reynolds, A., "An adaptive hierarchical multiscale algorithm for estimation of optimal well controls". SPE Journal, 19 (05), 2014, pp.909-930.

7. Suwartadi, E., Krogstad, S., Foss, B., "Adjoint-based surrogate optimization of oil reservoir water flooding". Optimization and Engineering, 16 (2), 2014, pp.441-481.

8. Humphries, T. D., Haynes, R. D., James, L. A., "Simultaneous and sequential approaches to joint optimization of well placement and control”. Computational Geosciences, 18 (3-4), 2013, pp.433-448.
9. Isebor, O. J., Ciaurri, D. E., Durlofsky, L. J., "Generalized fielddevelopment optimization with derivative-free procedures". SPE Journal, 19 (05), 2014, pp.891-908.

10. Tavallali, M. S., Karimi, I. A., Teo, K. M., Baxendale, D., Ayatollahi, S., "Optimal producer well placement and production planning in an oil reservoir". Computers \& Chemical Engineering, 55, 2013, pp.109-125.

11. Zhang, L., Zhang, K., Chen, Y., Li, M., Yao, J., Li, L., Lee, J., "Smart well pattern optimization using gradient algorithm". Journal of Energy Resources Technology, 138 (1), 2015, pp.012901.

12. Jansen, J. D., "Adjoint-based optimization of multi-phase flow through porous media-a review”. Computers \& Fluids, 46 (1), 2011, pp.40-51.

13. Luo, J., Qi, Y., Xie, J., Zhang, X., “A hybrid multi-objective PSOEDA algorithm for reservoir flood control operation". Applied Soft Computing, 34, 2015, pp.526-538.

14. Giuliani, C. M., Camponogara, E., "Derivative-free methods applied to daily production optimization of gas-lifted oil fields". Computers \& Chemical Engineering, 75, 2015, pp.60--64.

15. Vaz, A. I. F., Vicente, L. N., "PSwarm: a hybrid solver for linearly constrained global derivative-free optimization". Optimization Methods and Software, 24 (4-5), 2009, pp.669-685.

16. Vaz, A. I. F., Vicente, L. N., "A particle swarm pattern search method for bound constrained global optimization". Journal of Global Optimization, 39 (2), 2007, pp.197-219.

17. Rios, L. M., Sahinidis, N. V., "Derivative-free optimization: a review of algorithms and comparison of software implementations". Journal of Global Optimization, 56 (3), 2013, pp.1247-1293.

18. Yu, Q., "A method for calculating the reasonable and extreme well density in a water flooding sandstone reservoir". Petroleum Expoloration and Development, 4, 1986, pp.49-54.

19. Ahmed, T., "Reservoir engineering handbook (fourth edition)". Gulf Professional Publishing, United States, Boston, 2010. 